\title{
A Case Report on Epulis Fissuratum
}

\author{
Rubiya Parveen ${ }^{1}$, Veena A Patil ${ }^{2}$, Pranitha Surve ${ }^{3}$
}

\begin{abstract}
Aim: The aim of this case report was to diagnose an abnormal growth along a ill-fitting maxillary removable partial denture in a 60 -year-old woman and the surgical management of that lesion.

Background: Epulis fissuratum, reactive fibrous hyperplasia, or denture-induced fibrous hyperplasia are the various names attributed to a reactive tissue response to chronic irritation and trauma caused by a badly fitted prosthesis. Persistent trauma to oral mucosa may predispose the patient to a carcinoma. We present a case of faulty denture and the resultant epulis fissuratum in a 60-year-old woman.

Case description: A 60-year-old woman is presented to the Department of Periodontics with the chief complaint of an abnormal growth along the anterior border of her ill-fitting maxillary partial denture. An intraoral examination revealed a fibrous mass, which was polypoid and had a soft consistency and a smooth texture. The lesion was diagnosed as epulis fissuratum or a denture-induced hyperplasia and surgically managed the lesion with a scalpel and electrocautery. Postoperative instructions were given and a follow-up evaluation was done for 3 months.

Conclusion:To prevent the occurrence of the epulis fissuratum lesion is to perform standard prosthetic treatments on the patients and carry out the necessary maintenance procedures of the denture as soon as possible. The maintenance and hygiene-related instructions have to be given. Clinical significance: A poorly fitted prosthesis can give rise to many problems such as pain, discomfort in mastication, difficulty in speech, and epulis fissuratum. Epulis fissuratum is an tissue overgrowth in the mucobuccal fold, induced by chronic trauma from an ill-fitting prosthesis. In this case report, we discuss a case of epulis fissuratum in a 60-year-old woman and the surgical management of the lesion.

Keywords: Denture-induced hyperplasia, Epulis fissuratum, Removable partial denture.

Journal of Oral Health and Community Dentistry (2019): 10.5005/jp-journals-10062-0049
\end{abstract}

\section{INTRODUCTION}

Some of the oral mucosal lesions occur as a result of acute and chronic irritations caused by factors associated with dentures. These lesions are known as denture-related mucosal lesions. ${ }^{1}$ This entity most often occurs in middle-aged and older adults and commonly in women patients. It is reported in $5-10 \%$ of the jaws with a dental prosthesis. ${ }^{2} \mathrm{~A}$ long-term use of denture may culminate into reactive lesions of the oral mucosa. Epulis fissuratum is one such denture related to a oral mucosal lesion. Epulis fissuratum is also known as denture-induced hyperplasia, inflammatory fibrous hyperplasia, granuloma fissuratum, and denture epulis. ${ }^{3}$

Epulis fissuratum is a benign reactive hyperplastic condition of the fibrous connective tissue that occurs adjacent to the flanges of complete or partial ill-fitting dentures. These dentures usually have flanges longer than usual and give rise to mild and chronic irritation of the adjacent mucosa. ${ }^{1}$ The anterior portion of the jaws are affected most commonly than posterior areas. The size of this lesion can be less than $1 \mathrm{~cm}$ to massive lesions. When the offending denture is removed, a characteristic fissure bounded by hyperplastic soft tissue on both sides is seen. The chronic nature of the process means that discomfort is often not a prominent feature and therefore the patient may continue to wear the denture until hyperplastic lesions of considerable size develop. ${ }^{4}$

However, the lesion may be associated with pain and discomfort when ulceration occurs. It not only produces pain and discomfort, but negatively affects the mastication, esthetics, and overall well-being of the patient. ${ }^{5}$ Chronic trauma to the oral mucosa by the sharp edges of teeth or ragged borders of ill-fitting dentures have the potential to cause oral carcinoma. ${ }^{2}$ Hence, ill-fitting dentures and their sequelae should not be overlooked.
${ }^{1}$ Department of Periodontics and Oral Implantology,HKES's S. Nijalingappa Institute of Dental Sciences and Research, Gulbarga, Karnataka, India

${ }^{2}$ Department of Periodontics, HKES SN Dental College, Gulbarga, Karnataka, India

${ }^{3}$ Department of Periodontics, HKES's S. Nijalingappa Institute of Dental Sciences and Research, Gulbarga, Karnataka, India

Corresponding Author: Rubiya Parveen, Department of Periodontics and Oral Implantology, HKES's S. Nijalingappa Institute of Dental Sciences and Research, Gulbarga, Karnataka, India, Phone: +91 9740285899, e-mail: rubiyaparveenu@gmail.com

How to cite this article: Parveen R, Patil VA, et al. A Case Report on Epulis Fissuratum. J Oral Health Comm Dent 2019;13(2):59-61.

Source of support: Nil

Conflict of interest: None

\section{Case Description}

A 60-year-old woman attended at the Department of Periodontics with the chief complaint of an abnormal growth along the anterior border of her ill-fitting maxillary partial denture, which gradually grew in size over the past 6 months. The denture was fabricated about 2 year ago. The patient has been suffering from pain and discomfort during mastication for the past 3 months. The patient has not removed the denture for 6 months.

An intraoral examination revealed a fibrous mass, which was polypoid and had a soft consistency and a smooth texture (Fig. 1), sized about $2 \mathrm{~cm} \times 2.5 \mathrm{~cm}$ (Fig. 2). No relevant medical history, and she did not use any medications.

Differential diagnosis included irritation fibroma, leaflike denture fibroma, benign mesenchymal tumors, and minor salivary gland tumors. According to the patient's history as well as intraoral 


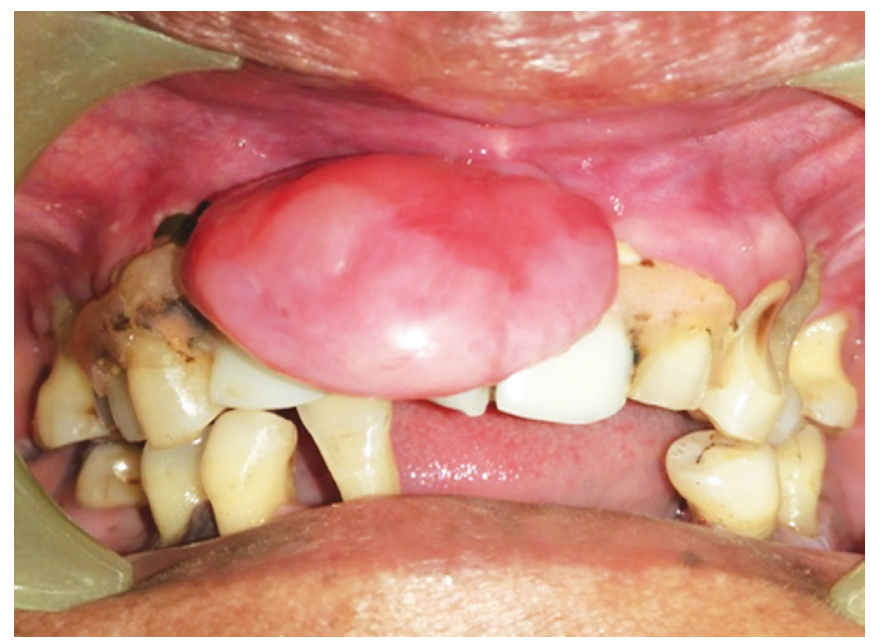

Fig. 1: Epulis fissuratum along the upper denture

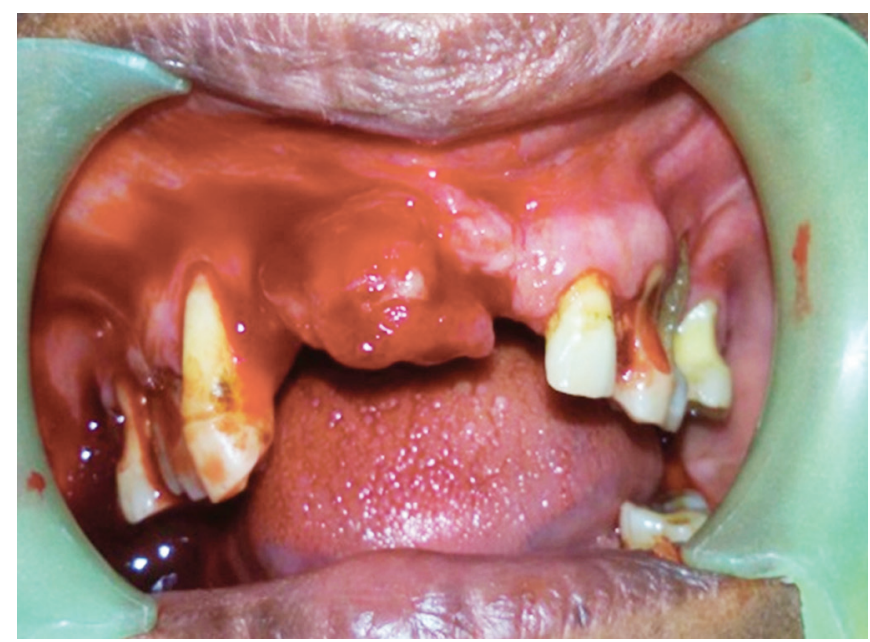

Fig. 3: Excision of lesion with a scalpel

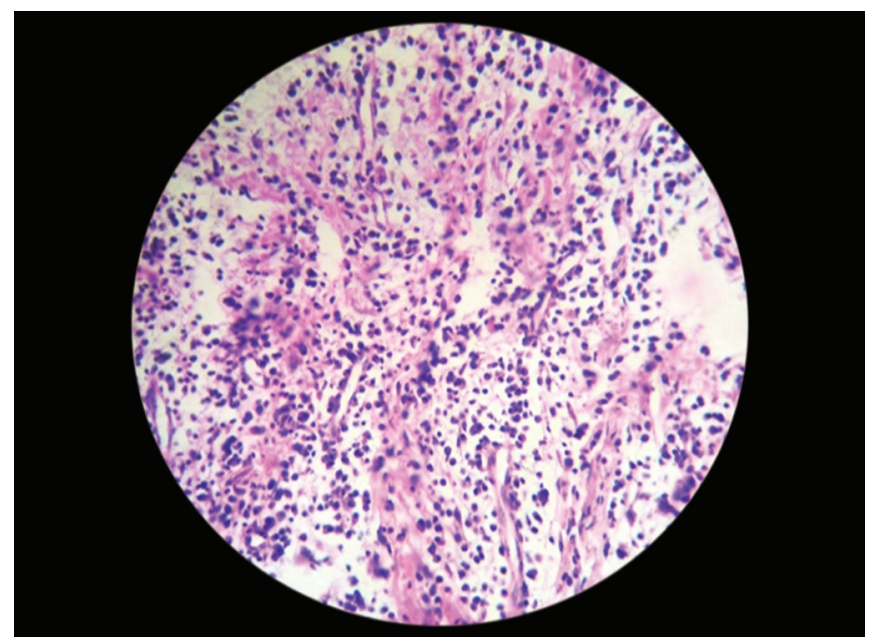

Fig. 5: Histopathological section showing an inflammatory cell infiltrate

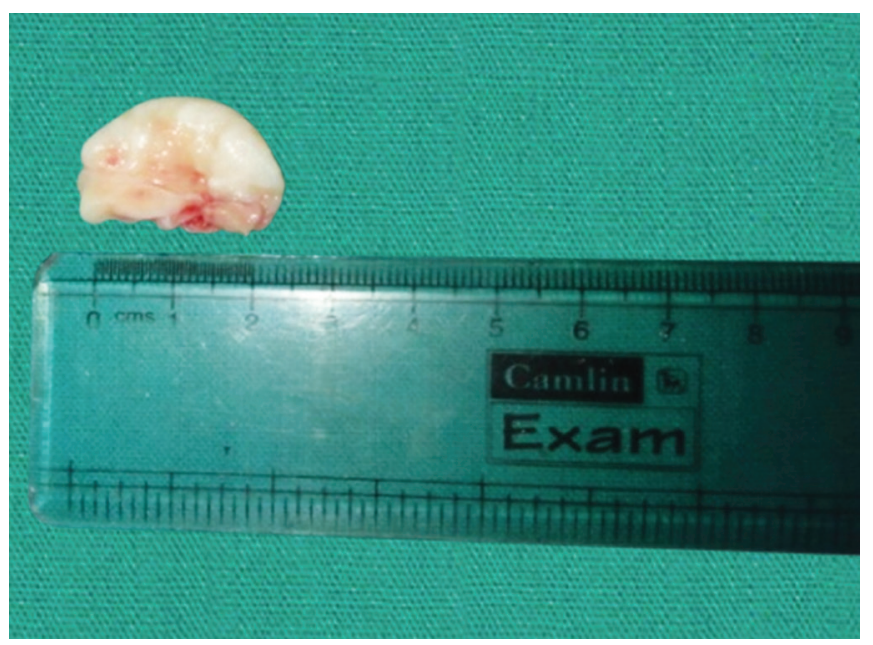

Fig. 2: Size of the lesion: $2 \mathrm{~cm}$

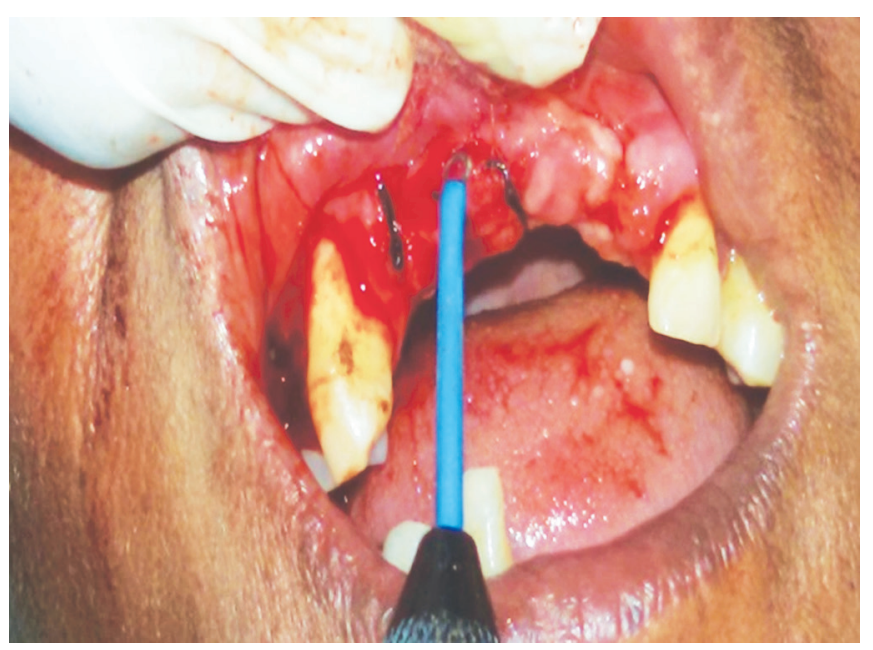

Fig. 4: Bleeding controlled by electrocautery

and extraoral clinical examination, a provisional diagnosis of a Denture-induced hyperplasia was made.

The treatment plan consisted of both medical and surgical approaches. The entire treatment plan was described to patient and written consent form was taken. The patient was instructed not to wear the denture. Nystatin oral suspension and ointment of triamcinolone were prescribed for her denture stomatitis. The patient was motivated and educated to maintain her oral hygiene.

After 10 days, the patient was recalled and oral prophylaxis was performed followed by a surgical excision of the lesion. A surgical procedure followed by isolation of the lesion and anesthesia of the area by bilateral anterior block was performed. With the help of a no. 15 scalpel blade, the lesion was excised from its base followed by suturing of the open edges (Fig. 3), and excessive bleeding was controlled by electrocautery (Fig. 4). Postoperative instructions were given and the specimen were collected and sent for histopathological examination, which showed hyperplastic epithelium as well as a fibrous connective tissue with moderate inflammation (Fig. 5). 


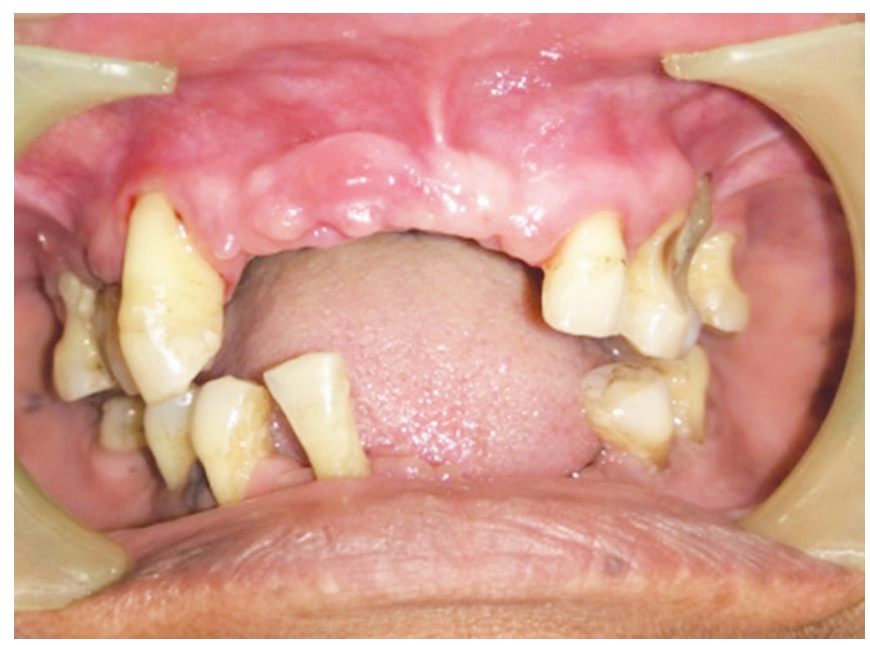

Fig. 6: Postoperative follow-up after 7 days

After 7 days, the patient was reexamined and the healing process was satisfactory (Fig. 6). Finally, the patient was referred to the department of prosthodontics for fabrication of the new denture. A 3 month regular follow-up showed complete resolution without any recurrence.

\section{Discussion}

The term "epulis" was first invented by Virchoft, and its dictionary meaning is "over the gum." Over the years, it was noticed that the usage of this term is not appropriate as it only refers to the site of the lesion. In most cases, the affected mucosa is usually the oral mucosa of the vestibular sulcus or the palatal region. Therefore, "denture-induced fibrous hyperplasia" was considered to be a much-preferred term. ${ }^{2}$

Epulis fissuratum or a denture-induced hyperplasia around the denture border is the result of the fibroepithelial response to denture wearing. It is often asymptomatic and may be limited to the tissues around the borders of the dentures in vestibular, lingual, or palatal regions. Epulis fissuratum occurs in the free mucosa lining the sulcus or at the junction of attached and free mucosa. With time, due to residual ridge resorption, even the best fitting denture gradually develop overextensions as a result of settling into different positions on a basal seat. Along with overextended borders, tipping forces resulting from imbalanced occlusion can also be a causative agent. $^{3}$

In an epidemiological survey, the incidence of epulis fissuratum was found to be 0.37 lesions per 1,000 persons in the 18-22 year age group and in people aged $>35$ years was found to be 4.1 per 1,000 persons and with an incidence of $3.5 \%$ in males and $4.4 \%$ in women. ${ }^{7}$ DIH may be the result of the ill-fitting denture, wearing denture all day and night long, poor oral hygiene, smoking, agerelated changes, and systemic conditions. ${ }^{2}$ In our case, ill-fitting denture and poor oral hygiene maintenance seemed to induce oral mucosal overgrowth. Firoozmand et al. showed that $78 \%$ of denture wearer women presented with a denture-induced hyperplasia mostly in the maxilla. ${ }^{2}$

In almost all previous reports, a denture-induced hyperplasia was demonstrated in the anterior portion of the mandible or maxilla. Histologically overlying epithelium is frequently hyperparakeratotic and demonstrates irregular hyperplasia of the rete ridges with underlying hyperplastic fibro-vascular connective tissue. A variable chronic inflammatory infiltrates was also be seen. ${ }^{7}$ The differential diagnosis includes peripheral giant cell granuloma, peripheral ossifying fibroma, bony exostosis and pyogenic granuloma, benign mesenchymal tumor, and minor salivary gland tumor. ${ }^{8}$ Treatment of epulis fissuratum may be conservative or surgical. A conservative approach should be considered as the first option because of its noninvasive nature. However, a conservative approach is timeconsuming and initially requires removal of the acrylic flange associated with the trauma and relining or repairing of the full denture. After a few weeks, when the lesion is completely healed, the acrylic flange may be relined and redesigned correctly to avoid further trauma of the mucosa when it is fitted in the mouth. ${ }^{6}$

Surgical methods mainly include removal of the lesion using blade, electrocauterization, or laser. Blade excision always requires infiltrated or general anesthesia and sutures or periodontal dressing. The necessity of infiltrated or general anesthetic is essential in electrosurgery but usually without sutures or periodontal dressing. ${ }^{6}$ In the present case, a surgical excision was done by a conventional surgical approach later on bleeding was controlled by electrocautery.

\section{Conclusion}

The modern dentistry requires the dentists to obtain the necessary knowledge in order to protect the health of the oral tissues and prevent the chronic diseases of the oral mucosa. One of the most important responsibilities of the dentists at the time the denture delivery to the patients is to provide specific instructions in order to protect the health of the mucosal tissues under the denture. The maintenance and oral hygiene-related instructions such as how to wash the denture and the length of wearing the denture throughout the day and also recommendations on regular visits to make sure the health of the oral tissues under the denture over time are among these necessary instructions.

\section{Acknowledgment}

The author personally thanks Dr Mrs Veena A Patil, Guide and HOD of the Department of Periodontics, HKES SN Dental College, Gulbarga for her untiring guidance during the treatment of the case.

\section{References}

1. Mohammadi M, Navabi N, et al. Clinical and denture-related characteristics in patients with epulis fissuratum: a retrospective 58 case series. Caspian J Dent Res 2017;6(1):15-21.

2. Mortazavi $\mathrm{H}$, Khalighi HR, et al. Epulis fissuratum in the soft palate: Report of a case in a very rare location. Dent Hypotheses 2016;7(2): 67.

3. Bhoyar A, Tijare M. An Unusual Occurrence of Epulis Fissuratum in Mandible; A Case Report and Literature Overview. Pariper-Indian Journal of Research 2016;5(10):335-337.

4. Veena KM, Jagadishchandra $H$, et al. An extensive denture-induced hyperplasia of the maxilla. Ann Med Health Sci Res 2013;3(1a):7-9. DOI: $10.4103 / 2141-9248.121208$.

5. Mohan RP, Verma S, et al. Epulis fissuratum: consequence of ill-fitting prosthesis. BMJ Case Rep 2013;2013:bcr2013200054. DOI: 10.1136/ bcr-2013-200054.

6. Kafas $P$, Upile T, et al. Mucogingival overgrowth in a geriatric patient. Dermatol Online J 2010;16(8):7.

7. Patil BA, Arora A, et al. Report of a Case of Epulis Fissuratum.Sch J Med Case Rep 2014;2(7):452-454.

8. Khan MW, Shah AA. A Massive Denture Induced Hyperplastic Lesion in Maxilla. J Pak Dent Assoc 2019;28(01):48. 